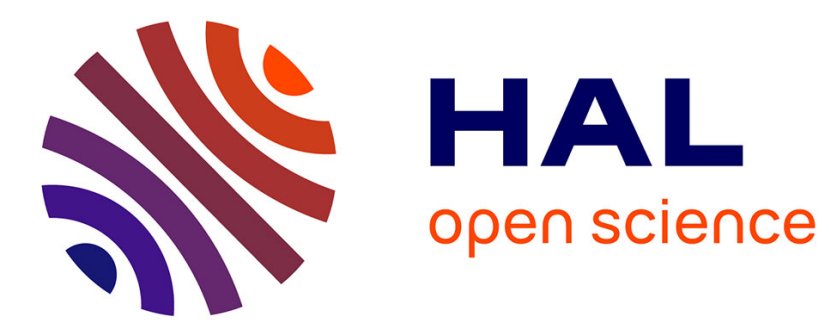

\title{
Frequency prediction from exact or self-consistent mean flows
}

\author{
Yacine Bengana, Laurette Tuckerman
}

\section{To cite this version:}

Yacine Bengana, Laurette Tuckerman. Frequency prediction from exact or self-consistent mean flows. Physical Review Fluids, 2021, 6 (6), 10.1103/PhysRevFluids.6.063901 . hal-03255327

\section{HAL Id: hal-03255327 \\ https://hal.science/hal-03255327}

Submitted on 9 Jun 2021

HAL is a multi-disciplinary open access archive for the deposit and dissemination of scientific research documents, whether they are published or not. The documents may come from teaching and research institutions in France or abroad, or from public or private research centers.
L'archive ouverte pluridisciplinaire HAL, est destinée au dépôt et à la diffusion de documents scientifiques de niveau recherche, publiés ou non, émanant des établissements d'enseignement et de recherche français ou étrangers, des laboratoires publics ou privés. 


\title{
Frequency prediction from exact or self-consistent mean flows
}

\author{
Yacine Bengana ${ }^{*} *$ \\ Department of Aeronautics, Imperial College London, South Kensington, London SW7 2AZ, United Kingdom \\ Laurette S. Tuckerman $\oplus^{\dagger}$ \\ Physique et Mécanique des Milieux Hétérogènes, CNRS, ESPCI Paris, Université PSL, Sorbonne Université, \\ Université de Paris, 75005 Paris, France
}

(Received 14 February 2021; accepted 20 May 2021; published 7 June 2021)

\begin{abstract}
A number of approximations have been proposed to estimate basic hydrodynamic quantities, in particular, the frequency of a limit cycle. One of these, real zero imaginary frequency (RZIF), calls for linearizing the governing equations about the mean flow and estimating the frequency as the imaginary part of the leading eigenvalue. A further reduction, the self-consistent model (SCM), approximates the mean flow as well, as resulting only from the nonlinear interaction of the leading eigenmode with itself. Both RZIF and SCM have proven dramatically successful for the archetypal case of the wake of a circular cylinder. Here, the SCM is applied to thermosolutal convection, for which a supercritical Hopf bifurcation gives rise to branches of standing waves and traveling waves. The SCM is solved by means of a full Newton method coupling the approximate mean flow and leading eigenmode. Although the RZIF property is verified for the traveling waves, the SCM reproduces the nonlinear frequency only very near the onset of the bifurcation and for another isolated parameter value. Thus, the nonlinear interaction arising from the leading mode is insufficient to reproduce the nonlinear mean field and frequency.
\end{abstract}

DOI: 10.1103/PhysRevFluids.6.063901

\section{INTRODUCTION}

Periodic emission or translation of vortical structures is one of the most important phenomena observed in hydrodynamic configurations. The amplitude and frequency are two essential characteristics of these time-periodic systems. These are usually obtained either by experiment or by solving the full Navier-Stokes equations by direct numerical simulation. The archetype of such configurations is the wake of a circular cylinder, in which the visually appealing Bénard-von-Kármán vortex "street" [1,2] appears above a Reynolds-number threshold [3,4] of 46.

When periodic oscillations such as these originate from a supercritical Hopf bifurcation, linear stability analysis about the equilibrium solution at the threshold yields a leading eigenvalue whose real part is zero and whose imaginary part is the oscillation frequency. Away from the threshold, this is no longer the case. However, for the cylinder wake, linearization about the time-averaged field has been shown to yield the nonlinear frequency [5-8] as the imaginary part of the leading eigenvalue. Moreover, Barkley [6] noted that the real part of this eigenvalue is nearly zero, meaning that the mean flow can be considered to be marginally stable, as had been suggested by Malkus [9] in the context of turbulent shear flow. This property, given the name real zero imaginary frequency (RZIF) by Turton et al. [10], has since been demonstrated to hold for several other flow

\footnotetext{
*b.y.bengana@gmail.com

†laurette.tuckerman@espci.fr
} 
configurations, namely, traveling waves in thermosolutal convection [10], spirals and ribbons in counter-rotating Taylor-Couette flow [11], and (approximately) for the flow in a two-dimensional shear-driven cavity [7,12].

We emphasize that the RZIF property is not universal for oscillating flows, since Turton et al. [10] have shown that the standing waves in thermosolutal convection emphatically do not satisfy this property. Nor is RZIF a prediction, since it relies on the mean flow that must be determined by experiment or direct numerical simulation. In contrast to RZIF, the self-consistent model (SCM) developed by Mantič-Lugo et al. [13] is predictive, or rather, it greatly reduces the computational work required to determine the frequency. In the SCM, the mean-flow equation is approximated by assuming that only the leading eigenmode of the linearized equation is responsible for creating the mean-flow distortion (the difference between the mean flow and the unstable equilibrium). This assumption is based on the fact that the temporal spectrum of the flow under investigation is dominated by its fundamental frequency. The amplitude of the mode corresponding to the fundamental frequency is chosen such that the growth rate of the linear problem is zero, thus building into the solution the "RZ" portion of the RZIF property. For the cylinder wake, the results obtained by these coupled equations match the mean flow and the nonlinear frequency remarkably well $[13,14]$. The SCM has also been used to treat acoustic emissions in the compressible wake of a cylinder [15] and the two-dimensional shear driven cavity [16]. Other reduced-order models in which sets of modes or interactions are omitted have been proposed and implemented for many other hydrodynamic phenomena, notably in aeronautics and fluid mechanics [17-34]. and in geophysics and astrophysics [35-40]. Some of these models will be compared to RZIF and SCM in the next section.

Here we investigate the self-consistent model for the traveling wave branch in thermosolutal convection, for which RZIF is satisfied [10]. We will demonstrate that, for this case, the selfconsistent model fails to predict the frequency or the mean flow. Higher-order terms contributing to the Reynolds stress are necessary to reproduce the mean flow to sufficient accuracy. Therefore, satisfaction of the RZIF property does not necessarily imply the validity of the self-consistent model.

\section{RZIF AND SCM FRAMEWORK}

We present in this section the equations governing the formalism of the RZIF and SCM approximations. Consider a general dynamical system of the form

$$
\partial_{t} U=\mathcal{L} U+\mathcal{N}(U, U)
$$

where $\mathcal{L}$ and $\mathcal{N}$ are linear and bilinear operators, respectively, and $U$ may depend on one or more spatial dimensions. The operators $\mathcal{L}$ and $\mathcal{N}$ depend on a control parameter $r$. We assume that Eq. (1) has an equilibrium (base) state $U_{\mathrm{b}}$ and undergoes a supercritical Hopf bifurcation at a critical value $r_{\text {Hopf }}$ leading to a stable limit cycle. The base state satisfies

$$
0=\mathcal{L} U_{\mathrm{b}}+\mathcal{N}\left(U_{\mathrm{b}}, U_{\mathrm{b}}\right)
$$

Classic linear stability analysis is derived by writing $U=U_{\mathrm{b}}+u$, substituting into Eq. (1):

$$
\partial_{t} u=\mathcal{L} U_{\mathrm{b}}+\mathcal{L} u+\mathcal{N}\left(U_{\mathrm{b}}, U_{\mathrm{b}}\right)+\mathcal{N}\left(u, U_{\mathrm{b}}\right)+\mathcal{N}\left(U_{\mathrm{b}}, u\right)+\mathcal{N}(u, u)
$$

subtracting Eq. (2):

$$
\partial_{t} u=\mathcal{L} u+\mathcal{N}\left(u, U_{\mathrm{b}}\right)+\mathcal{N}\left(U_{\mathrm{b}}, u\right)+\mathcal{N}(u, u),
$$

and neglecting the nonlinear terms $\mathcal{N}(u, u)$ :

$$
\partial_{t} u=\mathcal{L} u+\mathcal{N}\left(u, U_{\mathrm{b}}\right)+\mathcal{N}\left(U_{\mathrm{b}}, u\right) .
$$

Since Eq. (5) is linear in $u$ and homogeneous in time, its solution is of the form $u(t)=\exp \left[\left(\sigma_{\mathrm{b}}+\right.\right.$ $\left.\left.i \omega_{\mathrm{b}}\right) t\right] u_{\mathrm{b}}$ with

$$
\left(\sigma_{\mathrm{b}}+i \omega_{\mathrm{b}}\right) u_{\mathrm{b}}=\mathcal{L}_{U_{\mathrm{b}}} u_{\mathrm{b}},
$$


where we have defined

$$
\mathcal{L}_{U_{\mathrm{b}}} \equiv \mathcal{L}+\mathcal{N}\left(U_{\mathrm{b}}, \cdot\right)+\mathcal{N}\left(\cdot, U_{\mathrm{b}}\right) .
$$

Like $\mathcal{L}$ and $U_{\mathrm{b}}$, the eigenvalue $\sigma_{\mathrm{b}}+i \omega_{\mathrm{b}}$ depends on the parameter $r$. When the growth rate $\sigma_{\mathrm{b}}$ crosses zero at $r=r_{\text {Hopf }}$ and $\omega_{\mathrm{b}} \neq 0$, the base state $U_{\mathrm{b}}$ undergoes a supercritical Hopf bifurcation, creating a new limit cycle $U_{\mathrm{lc}}(t)$, satisfying

$$
\partial_{t} U_{\mathrm{lc}}(t)=\mathcal{L} U_{\mathrm{lc}}(t)+\mathcal{N}\left[U_{\mathrm{lc}}(t), U_{\mathrm{lc}}(t)\right],
$$

and whose frequency is $\omega_{\mathrm{b}}$ at onset. For $r$ beyond $r_{\mathrm{Hopf}}$, the frequency $\omega_{\mathrm{lc}}$ of the limit cycle is no longer equal to $\omega_{\mathrm{b}}$.

We now consider the temporal mean $\bar{U}$ of the limit cycle $U_{\mathrm{lc}}(t)$ :

$$
\bar{U} \equiv \frac{1}{T_{\mathrm{lc}}} \int_{t=0}^{T_{\mathrm{lc}}} U_{\mathrm{lc}}(t) d t,
$$

where $T_{\mathrm{lc}}=2 \pi / \omega_{\mathrm{lc}}$. Substituting the Reynolds decomposition $U=\bar{U}+u$ into the governing Eq. (1), we obtain

$$
\partial_{t} u=\mathcal{L} \bar{U}+\mathcal{L} u+\mathcal{N}(\bar{U}, \bar{U})+\mathcal{N}(u, \bar{U})+\mathcal{N}(\bar{U}, u)+\mathcal{N}(u, u) .
$$

The temporal average of Eq. (9) gives the equations obeyed by the mean fields

$$
0=\mathcal{L} \bar{U}+\mathcal{N}(\bar{U}, \bar{U})+\overline{\mathcal{N}}(u, u),
$$

where the nonlinear interaction term $\overline{\mathcal{N}(u, u)}$ is the force resulting from what is called the Reynolds stress in the context of hydrodynamics. It can also be viewed as the external force that would be required for the mean field to be a stationary solution [6]. The mean field is computed from nonlinear simulations because Eq. (10), unlike Eq. (2), is not a closed system. By subtracting Eq. (10) from Eq. (9), we obtain the exact fluctuation equation:

$$
\partial_{t} u=\underbrace{\mathcal{L} u+\mathcal{N}(u, \bar{U})+\mathcal{N}(\bar{U}, u)}_{\mathcal{L}_{\bar{U}} u}+\underbrace{\mathcal{N}(u, u)-\overline{\mathcal{N}(u, u)}}_{g} .
$$

\section{A. RZIF}

The RZIF procedure calls for omitting the nonlinear terms $g$ from Eq. (11). This omission is exact if the nonlinear self interaction $\mathcal{N}(u, u)$ of the deviation $u$ from the mean contributes only to the mean. (We will discuss this point further in Sec. IV.) This leaves

$$
\partial_{t} u=\mathcal{L}_{\bar{U}} u \equiv \mathcal{L} u+\mathcal{N}(u, \bar{U})+\mathcal{N}(\bar{U}, u) .
$$

Since Eq. (12) is linear in $u$ and homogeneous in $t$, its solutions are again of the form $u(t)=$ $\exp \left[\left(\sigma_{\text {rzif }}+i \omega_{\text {rzif }}\right) t\right] u_{\text {rzif }}$, leading again to the eigenproblem

$$
\left(\sigma_{\text {rzif }}+i \omega_{\text {rzif }}\right) u_{\text {rzif }}=\mathcal{L}_{\bar{U}} u_{\text {rzif }} .
$$

Limit cycles satisfy the RZIF property if the imaginary part $\omega_{\text {rzif }}$ of the leading eigenmode of $\mathcal{L}_{\bar{U}}$ is equal to the frequency $\omega_{\mathrm{lc}}$ of the nonlinear limit cycle $U_{\mathrm{lc}}(t)$ and the real part $\sigma_{\text {rzif }}$ is zero. Since $g$ in Eq. (11) is exactly zero only under special circumstances, RZIF will typically be satisfied only approximately. Equations (10) and (12) comprise the linearization about the mean fields studied in Refs. [5-8,10-12]

\section{B. SCM}

The RZIF Eqs. (10) and (13) are not predictive or closed, because the mean flow $\bar{U}$ must be computed in some other way, sometimes from experimental data but more often by time averaging the results of a full direct numerical simulation of the limit cycle. In contrast, the SCM developed by Mantič-Lugo et al. [13] does not require the mean flow as an input. Instead, these authors make 
TABLE I. Specification of classic linear stability analysis about the base flow (LSA), linearization about the mean (RZIF), and the self-consistent model (SCM). The equations in the column labeled System define the problem, while the equations in the column labeled Property may or may not be satisfied by the corresponding system, or may be satisfied only approximately.

\begin{tabular}{|c|c|c|c|c|}
\hline & Name & Linearize about & System & Property \\
\hline LSA & Linear stability analysis & Base flow $U_{\mathrm{b}}$ & $\begin{array}{l}0=\mathcal{L} U_{\mathrm{b}}+\mathcal{N}\left(U_{\mathrm{b}}, U_{\mathrm{b}}\right) \\
\left(\sigma_{\mathrm{b}}+i \omega_{\mathrm{b}}\right) u_{\mathrm{b}}=\mathcal{L}_{U_{\mathrm{b}}} u_{\mathrm{b}}\end{array}$ & \\
\hline \multirow[t]{2}{*}{ RZIF } & Real zero & Mean flow $\bar{U}$ & $\partial_{t} U_{\mathrm{lc}}=\mathcal{L} U_{\mathrm{lc}}+\mathcal{N}\left(U_{\mathrm{lc}}, U_{\mathrm{lc}}\right)$ & \\
\hline & imaginary frequency & & $\begin{array}{c}U_{\mathrm{lc}}\left(T_{\mathrm{lc}}\right)=U_{\mathrm{lc}}(0) \quad \bar{U} \equiv \frac{1}{T_{\mathrm{lc}}} \int_{0}^{T_{\mathrm{lc}}} U_{\mathrm{lc}}(t) d t \\
\left(\sigma_{\mathrm{rzif}}+i \omega_{\mathrm{rzif}}\right) u_{\mathrm{rzzi}}=\mathcal{L}_{\bar{U}} u_{\mathrm{rzif}}\end{array}$ & $\begin{array}{c}\omega_{\text {rzif }}=\omega_{\mathrm{lc}} \\
\sigma_{\text {rzif }}=0\end{array}$ \\
\hline SCM & Self-consistent model & $\begin{array}{l}\text { Approximate } \\
\text { mean flow } U_{\mathrm{scm}}\end{array}$ & $\begin{array}{c}0=\mathcal{L} U_{\mathrm{scm}}+\mathcal{N}\left(U_{\mathrm{scm}}, U_{\mathrm{scm}}\right)+\mathcal{N}\left(u_{\mathrm{scm}}, u_{\mathrm{scm}}^{*}\right) \\
i \omega_{\mathrm{scm}} u_{\mathrm{scm}}=\mathcal{L}_{U_{\mathrm{scm}}} u_{\mathrm{scm}}\end{array}$ & $\omega_{\mathrm{scm}}=\omega_{\mathrm{lc}}$ \\
\hline
\end{tabular}

the further hypothesis that the contribution from the leading eigenmode suffices to generate the mean-flow distortion, i.e., its deviation from the base flow. According to this approximation, $u$ in $\overline{\mathcal{N}(u, u)}$ in Eq. (10) is no longer the deviation from the mean of the limit cycle, but an eigenvector $u_{\mathrm{scm}}$. Moreover, they hypothesize that $u_{\mathrm{scm}}$ can be chosen (via its amplitude; see Sec. VI) such that the real part of the eigenvalue is zero, i.e. such that $U_{\mathrm{scm}}$ is marginally stable. This leads to the following problem:

$$
\begin{aligned}
0 & =\mathcal{L} U_{\mathrm{scm}}+\mathcal{N}\left(U_{\mathrm{scm}}, U_{\mathrm{scm}}\right)+\mathcal{N}\left(u_{\mathrm{scm}}, u_{\mathrm{scm}}^{*}\right) \\
i \omega_{\mathrm{scm}} u_{\mathrm{scm}} & =\mathcal{L}_{U_{\mathrm{scm}}} u_{\mathrm{scm}}
\end{aligned}
$$

where

$$
\mathcal{L}_{U_{\mathrm{scm}}} \equiv \mathcal{L}+\mathcal{N}\left(\cdot, U_{\mathrm{scm}}\right)+\mathcal{N}\left(U_{\mathrm{scm}}, \cdot\right) .
$$

Table I summarizes the linear stability problem and the RZIF and SCM approximations.

\section{Semilinear or quasilinear models}

To place RZIF and SCM in context, these are variants of a large family of approximations based on partitioning the velocity field into two components, $\bar{U}$ and $u . \bar{U}$ varies, if at all, only on large spatial or temporal scales, while $u$ is governed by an equation that depends on $\bar{U}$ and is linear in $u$. The equation for $\bar{U}$ contains nonlinear terms in $u$ which influence $\bar{U}$; for the Navier-Stokes equations, these are the quadratic terms arising from the Reynolds stress. Nonlinear terms in $u$ which do not contribute to $\bar{U}$ are omitted.

Such approximations can be classified according to the type of partition, i.e., what defines the set $\bar{U}$ and $u$. The RZIF and SCM approximations partition in the temporal frequency domain. $\bar{U}$ is the temporal mean and $u$ the time-varying field. Since $\bar{U}$ is the temporal mean, it is constant, and since $u$ satisfies a linear equation, it is an eigenvector. These approximations are therefore not suitable for time integration. Instead, they have been used to determine the frequency and to approximate the spatiotemporal form of a limit cycle.

McKeon and Sharma [17] proposed a temporal partition approach in which $g \equiv \mathcal{N}(u, u)-$ $\overline{\mathcal{N}(u, u)}$ in Eq. (11) is not omitted but instead considered as an input to the transfer function or resolvent operator $\left(i \omega-\mathcal{L}_{\bar{U}}\right)^{-1}$. Note that if Eq. (13) holds with $\sigma=0$, then $\left(i \omega-\mathcal{L}_{\bar{U}}\right)$ has a nontrivial kernel and is therefore noninvertible. In the resolvent approach, $\left(i \omega-\mathcal{L}_{\bar{U}}\right)$ is considered to be invertible but to have one or a few singular values much smaller than the others. The resolvent $\left(i \omega-\mathcal{L}_{\bar{U}}\right)^{-1}$ then acts as a filter by highly amplifying the component(s) in $g$ of the corresponding singular vector(s). The resolvent is often studied in the context of the optimal forcing problem, that 
of determining the forcing function and frequency which are maximally amplified. In its most basic form, this problem is

$$
\begin{aligned}
0 & =\mathcal{L} \bar{U}+\mathcal{N}(\bar{U}, \bar{U})+\overline{\mathcal{N}(u, u)}, \\
\left(i \omega-\mathcal{L}_{\bar{U}}\right) u & =f e^{i \omega t} .
\end{aligned}
$$

As in the distinction between RZIF and SCM, two variants are possible: Eq. (15b) can be solved on its own using the exact mean $\bar{U}$, or it can use the $\bar{U}$ calculated self-consistently by the coupled system Eqs. (15a) and (15b). The nonlinear optimal forcing problem is more exact than the linear optimal forcing problem, since it retains in (15b) the nonlinear terms $g$ defined in (11) as well as the imposed forcing function $f$. The resolvent and generalizations of it have been used in Refs. [17-27] to approximate the optimal forcing or the energy spectrum of complex and even turbulent flows.

A complementary approach partitions the spatial, rather than temporal, dependence of solutions into a spatial mean $\langle U\rangle$ and spatially varying perturbations $u$. These approximations are sometimes called quasilinear (QL) models. Like projections of the governing equations onto a set of spatial basis functions, they can be integrated in time in the same way as the original equations:

$$
\begin{aligned}
\partial_{t}\langle U\rangle & =\mathcal{L}\langle U\rangle+\mathcal{N}(\langle U\rangle,\langle U\rangle)+\langle\mathcal{N}(u, u)\rangle, \\
\partial_{t} u & =\mathcal{L}_{\langle U\rangle} u
\end{aligned}
$$

where \langle\rangle is a spatial average.

One example of a spatial partition is the restricted nonlinear (RNL) model used by fluiddynamical researchers to study wall-bounded shear flows, in which $\langle U\rangle$ and $u$ are set to be the streamwise-averaged and streamwise-varying modes [29-31]. This model has reproduced many features of transitional and turbulent pipe flow and plane Couette flow. A similar approach is used in Ref. [32] to study the centrifugal instability on a vortex. One important current of research interprets transition to turbulence in wall-bounded shear flows as a skeleton of trajectories connecting steady states, traveling waves and periodic orbits, and other low-dimensional invariant dynamical objects, called exact coherent structures (ECS) in this context. These have been computed using the full Navier-Stokes equations, and successfully approximated via the RNL model in Refs. [33,34].

QL models have been widely used in the geophysical and astrophysical community. Marston et al. [35] have generalized this approach to the generalized quasilinear (GQL) approximation. In the GQL, a larger set of modes (usually those with low wave number) is treated in the same way as the mean, by including all nonlinear interactions involving this set, and excluding nonlinear interactions within the remaining (usually high wave number) modes that do not contribute to the low wave-number set. The QL and GQL approximations have been used to calculate the east-west bands or jets on planetary surfaces [35-38]. A study of rotating plane Couette flow [39] has provided an illustration of the ability of GQL to capture features that QL does not. Another type of mean flow for which the quasilinear approach can be used is the ensemble average [40]. Ensemble averaging, like temporal averaging, can also be combined with averaging over a homogeneous spatial direction as in Refs. [17,27].

Neither RZIF nor SCM fall precisely into the category of QL or GQL methods; see Sec. V.

\section{APPLICATION TO THERMOSOLUTAL WAVES}

We now turn to the hydrodynamic system for which we will compare RZIF and SCM. A density gradient in a fluid layer often leads to convection, i.e., overturning motion that tends to equalize the density in the bulk. The density gradient is in turn usually the consequence of thermal and/or concentration gradients; when both are present, terms such as thermosolutal, double-diffusive, and binary are used for different variants of the problem. If the thermal and solutal effects oppose one another, then convection can take the form of time-dependent solutions.

The thermosolutal problem studied here and in Refs. [10,41] is formulated in an idealized two-dimensional horizontally periodic domain $(x, z) \in[0,2.8) \times[0,1]$, allowing the velocity to be 

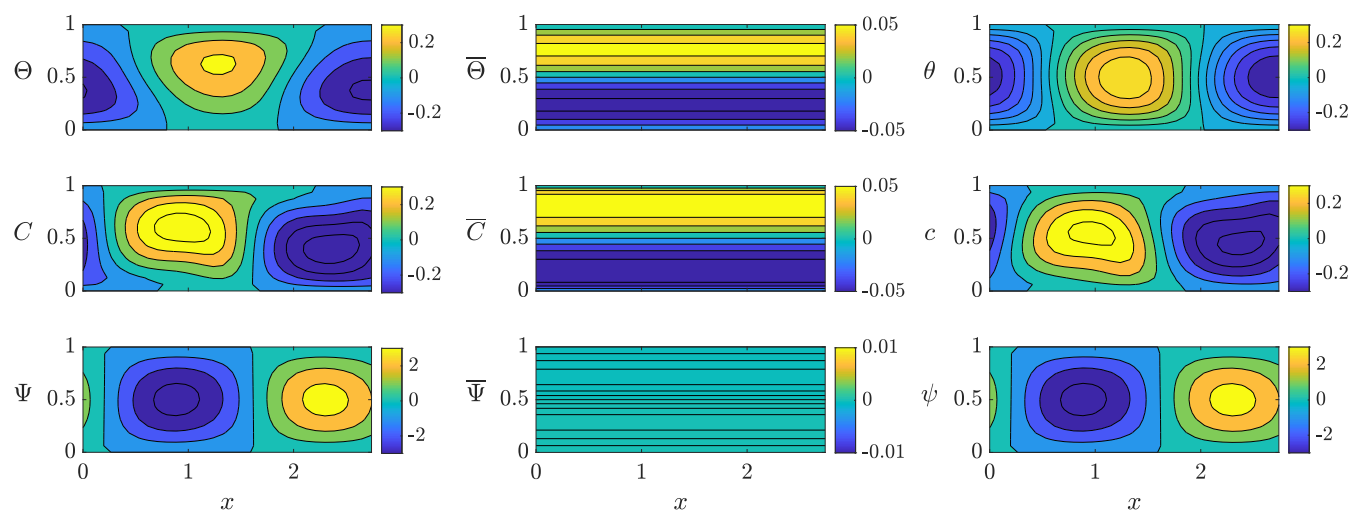

FIG. 1. Left: instantaneous snapshot $(\Theta, C, \Psi)$ of a traveling wave. $\Theta$ and $C$ are the deviation of the temperature and concentration from the linear conductive solution, and $\Psi$ is the stream function representing the velocity. Center: temporal mean flow $(\bar{\Theta}, \bar{C}, \bar{\Psi})$. Right: deviation $(\theta, c, \psi)$ from the mean flow. The mean field $(\bar{\Theta}, \bar{C}, \bar{\Psi})$ is much smaller than the instantaneous field, so the deviation $(\theta, c, \psi)$ is very close to the instantaneous field.

represented as $\nabla \times \Psi \mathbf{e}_{\mathbf{y}}$ and the equations to be stated in stream-function-vorticity form. At the top and bottom boundaries $z=0,1$, different values are imposed for the temperature and concentration, and free-slip conditions are imposed on the velocity. There exists a motionless conductive solution in which the temperature and concentration fields are linear functions of the vertical coordinate $z$. We set $\Theta$ and $C$ to be deviations of the temperature and concentration fields from the conductive profiles.

The nondimensionalized governing equations are

$$
\begin{aligned}
\partial_{t} \Theta-\mathcal{J}[\Psi, \Theta] & =\nabla^{2} \Theta+\partial_{x} \Psi, \\
\partial_{t} C-\mathcal{J}[\Psi, C] & =L \nabla^{2} C+\partial_{x} \Psi, \\
\partial_{t} \nabla^{2} \Psi-\mathcal{J}\left[\Psi, \nabla^{2} \Psi\right] & =P\left[\nabla^{4} \Psi+R_{T} \partial_{x}(\Theta+S C)\right],
\end{aligned}
$$

where the Poisson bracket is

$$
\mathcal{J}[f, g] \equiv \mathbf{e}_{\mathbf{y}} \cdot \nabla f \times \nabla g=\partial_{z} f \partial_{x} g-\partial_{x} f \partial_{z} g
$$

The ratio $P$ of kinematic viscosity to thermal diffusivity is fixed at 10 and the ratio $L$ of solutal to thermal diffusivity to 0.1 (these are the usual Prandtl number and inverse of the Lewis number, respectively). The imposed concentration and thermal gradients both contribute to the density gradient and the ratio $S$ of their contributions is fixed at -0.5 . We vary the imposed thermal gradient, which is given in terms of the reduced Rayleigh number $r$, the ratio of the Rayleigh number $R_{T}$ to its critical value 657.5 for this geometry and in the absence of a concentration gradient. The conductive solution is stable for $r$ until $r=2.05$, when a Hopf bifurcation breaks the translational symmetry in this periodic geometry, leading to the creation of branches of traveling and standing waves [42]. We carry out our study over the range $r \in[2.06,3]$.

Figure 1 shows an instantaneous visualisation in the $(x, z)$ plane of the exact nonlinear traveling wave $U_{\mathrm{lc}}$ and its decomposition into the temporal mean flow $\bar{U}$ and deviation $U_{\mathrm{lc}}-\bar{U}$. We emphasize that $\bar{U}$ is not the conductive solution, but the mean of the deviation from it, sometimes called the distortion. Because $U_{\mathrm{lc}}$ is a traveling wave, fields at other instants in time can be obtained by a shift in the periodic direction $x$, and the temporal mean is also the spatial mean in $x$. A detailed study of the RZIF property in traveling and standing waves in thermosolutal convection was carried out in Ref. [10]. Turton et al. [10] showed that the traveling waves had the RZIF property while standing waves at the same parameter values did not. We will extend the study of the thermosolutal 

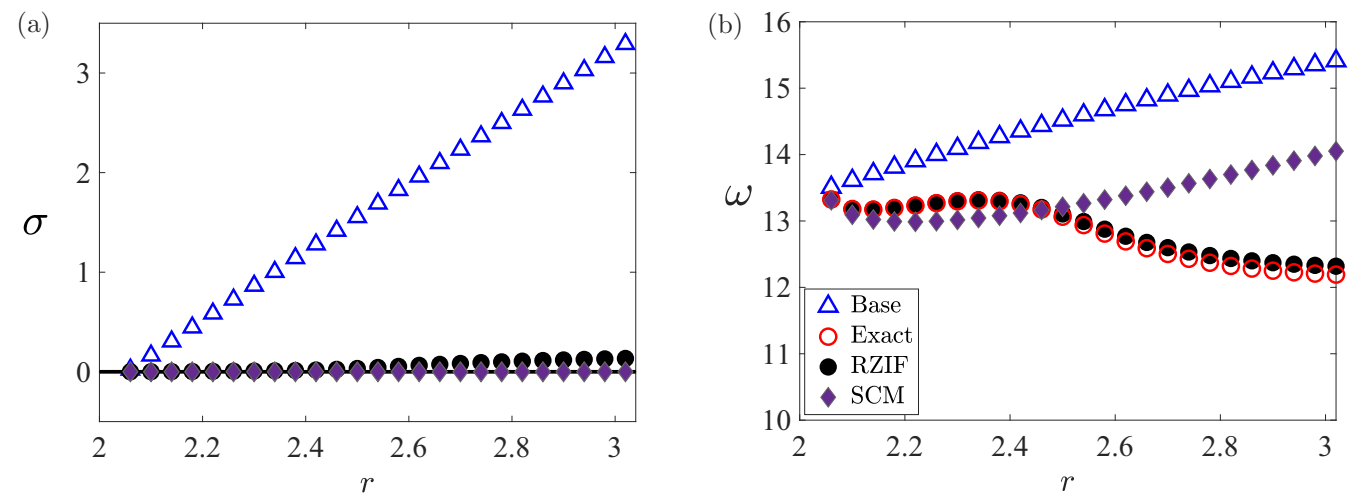

FIG. 2. (a) Growth rate and (b) frequency as a function of Rayleigh number. Exact frequencies are shown by open circles (o). Frequencies and growth rates obtained by linearization about the conductive base state are represented by triangles $(\triangle)$ while those obtained by linearization about the full mean field (RZIF procedure) are represented by solid circles $(\bullet)$. Frequencies obtained by the SCM procedure are shown by diamonds $(\diamond)$.

traveling waves to the SCM approximation. We do not include the standing waves, since the SCM approximation presupposes the validity of the RZIF approximation.

The main result of this study is contained in Fig. 2, which shows the exact frequency $\omega_{\mathrm{lc}}$ of the limit cycle, along with the real and imaginary parts of the eigenvalues of the operators $\mathcal{L}_{U_{\mathrm{b}}}$, $\mathcal{L}_{\bar{U}}$, and $\mathcal{L}_{U_{\text {scm }}}$ as a function of $r$. The frequency $\omega_{\mathrm{b}}$ obtained from linear stability analysis about the conductive base state is far from the frequency $\omega_{\mathrm{lc}}$ of the limit cycle, as expected, while the frequency $\omega_{\text {rzif }}$ obtained by RZIF, i.e., linearizing around the mean flow $\bar{U}$, is quite close to the exact nonlinear frequency and $\sigma_{\text {rzif }}$ remains small in the entire range investigated, [2.05,3]. In contrast, the frequency $\omega_{\text {scm }}$ obtained by SCM matches $\omega_{\text {lc }}$ only very close to the threshold, approximately for $r \in[2.05,2.08]$ and deviates below it for $r \geqslant 2.1$. However, as $r$ is increased further, the $\omega_{\text {scm }}$ curve approaches the $\omega_{\mathrm{lc}}$ curve, crossing it at $r=2.5$ and then exceeding it substantially. For $r \geqslant 2.5$, the RZIF growth rate $\sigma_{\text {rzif }}$ becomes slightly positive. The SCM growth rate $\sigma_{\text {scm }}$ is zero for all $r$ by construction.

In Fig. 3, we compare the mean concentration profile calculated by the SCM to the exact mean profile for various values of $r$. (Recall that the RZIF procedure uses the exact mean profile.) We choose the concentration, here and elsewhere, because the differences are largest for this component. The disagreement between the SCM and exact profiles closely follows the tendency seen in Fig. 2: a disagreement at $r=2.3$, which decreases to the point of being almost negligible at $r=2.5$ and then increases again with $r$. We also note that the sign of the error in the mean flows reverses at $r=2.5$,
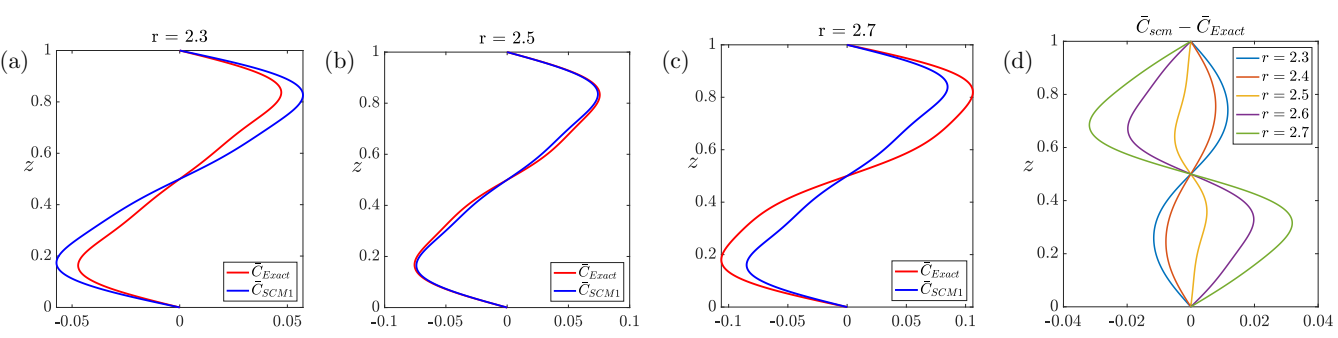

FIG. 3. Mean concentration profile calculated via SCM compared to the exact profile. (a) For $r=2.3$, the amplitude of $\bar{C}_{\mathrm{SCM}}$ is greater than that of $\bar{C}_{\text {exact }}$. (b) For $r=2.5$, the value at which $\omega_{\mathrm{scm}} \approx \omega_{\mathrm{lc}}$, the two profiles are almost identical. (c) For $r=2.7$, the amplitude of $\bar{C}_{\text {scm }}$ is less than that of $\bar{C}_{\text {Exact }}$. (d) Difference $\bar{C}_{\text {scm }}-\bar{C}_{\text {Exact }}$ for $2.3 \leqslant r \leqslant 2.7$. 
just as was seen for the frequency in Fig. 2. Thus, the crossing of $\omega_{\mathrm{scm}}$ and $\omega_{\mathrm{lc}}$ seen in Fig. 2 at $r=$ 2.5 is not a coincidence, e.g. two different operators sharing the same eigenvalues. The agreement between the eigenvalues at $r=2.5$ is due precisely to the fact that the SCM approximation to the mean field is accurate at that particular value.

This case provides a counterexample to the SCM, showing that the RZIF property does not necessarily imply the validity of SCM. The assumption that only the leading mode contributes significantly to the distortion of the mean field does not hold.

Mantič-Lugo and Gallaire [21] have carried out a study of the optimal forcing response in the backward facing step, comparing fully nonlinear results [retaining in (15b) the nonlinear terms $g$ of Eq. (11)] with linear results from the resolvent Eq. (15b), either computed from the exact mean flow or from a self-consistent approximation to the mean using a single mode as in Eq. (15a). Surprisingly, they find that the results from the single-mode approximation to the mean and resolvent (comparable to SCM) are much closer to the nonlinear results than those using the exact mean and resolvent (comparable to RZIF). This could be due to the consistency of the truncation used in $\mathrm{SCM}$, or to some difference between limit cycles and optimal forcing, or between the thermosolutal problem and the backward-facing step, or merely to chance.

\section{FOURIER ANALYSIS: HARMONIC BALANCE}

To further understand the RZIF and SCM equations, we turn to the temporal Fourier decomposition of the limit cycle and of the governing equations. The statement of the governing equations in terms of the temporal Fourier decomposition is called harmonic balance in the aerodynamic literature [27,43-45] and it is the basis of the argument presented in Turton et al. [10]. We write the limit cycle $U_{\mathrm{lc}}(t)$ as

$$
U_{\mathrm{lc}}=\bar{U}+\sum_{n \neq 0} \hat{u}_{n} e^{i n \omega t}
$$

where $\hat{u}_{-n}=\hat{u}_{n}^{*}$. Figure 4 shows these Fourier components for our case of traveling waves in thermosolutal convection. Their spatial form is dictated by the fact that a temporal Fourier decomposition is equivalent to a horizontal spatial Fourier decomposition for a traveling wave.

We then substitute Eq. (19) into the governing Eq. (1) and separate the resulting terms of different frequencies. The term corresponding to $n=0$ is the governing equation of the mean field:

$$
0=\mathcal{L} \bar{U}+\mathcal{N}(\bar{U}, \bar{U})+\underbrace{\sum_{m \neq 0} \mathcal{N}\left(\hat{u}_{m}, \hat{u}_{-m}\right)}_{\mathcal{N}_{0}} .
$$

The nonlinear term $\mathcal{N}_{0}$ appearing in Eq. (20a) is the divergence of the Reynolds stress, responsible for the distortion and production of the mean field. The equation corresponding to each $n>0$ is

$$
\operatorname{in} \omega \hat{u}_{n}=\underbrace{\mathcal{L} \hat{u}_{n}+\mathcal{N}\left(\bar{U}, \hat{u}_{n}\right)+\mathcal{N}\left(\hat{u}_{n}, \bar{U}\right)}_{\mathcal{L}_{\bar{U}} \hat{u}_{n}}+\underbrace{\sum_{m \neq 0, n} \mathcal{N}\left(\hat{u}_{m}, \hat{u}_{n-m}\right)}_{\mathcal{N}_{n}} .
$$

For $n=1$, Eq. (20b) becomes

where

$$
i \omega \hat{u}_{1}=\mathcal{L}_{\bar{U}} \hat{u}_{1}+\mathcal{N}_{1},
$$

$$
\mathcal{N}_{1} \equiv \mathcal{N}\left(\hat{u}_{2}, \hat{u}_{-1}\right)+\mathcal{N}\left(\hat{u}_{-1}, \hat{u}_{2}\right)+\mathcal{N}\left(\hat{u}_{3}, \hat{u}_{-2}\right)+\mathcal{N}\left(\hat{u}_{-2}, \hat{u}_{3}\right)+\ldots
$$

If the periodic cycle is exactly monochromatic, i.e., if $\hat{u}_{ \pm 2}=\hat{u}_{ \pm 3}=\ldots=0$, then $\mathcal{N}_{1}=0$ and Eq. (21) becomes the RZIF Eq. (13) with $\sigma_{\text {rzif }}=0$ :

$$
i \omega \hat{u}_{1}=\mathcal{L}_{\bar{U}} \hat{u}_{1} .
$$




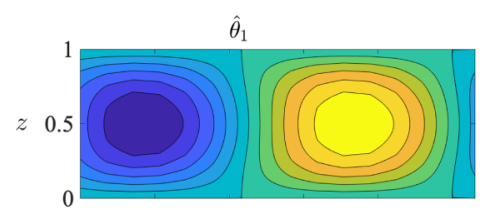

$\hat{c}_{1}$

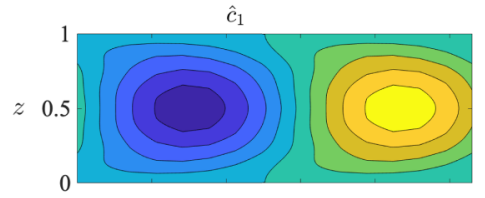

$\hat{\psi}_{1}$

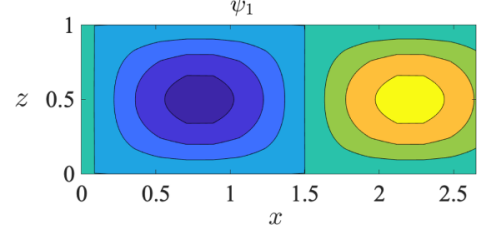

$\hat{\theta}_{2}$

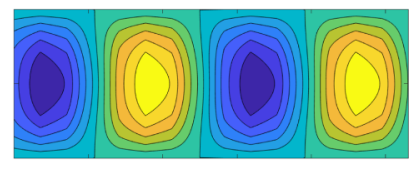

$\hat{c}_{2}$

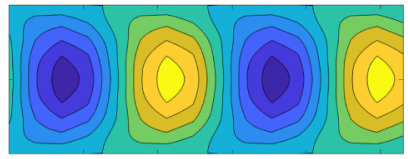

$\hat{\psi}_{2}$

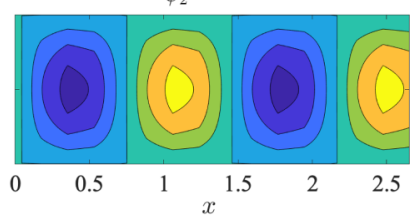

$\hat{\theta}_{3}$

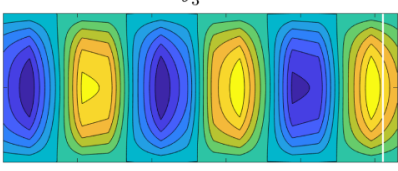

$\hat{c}_{3}$
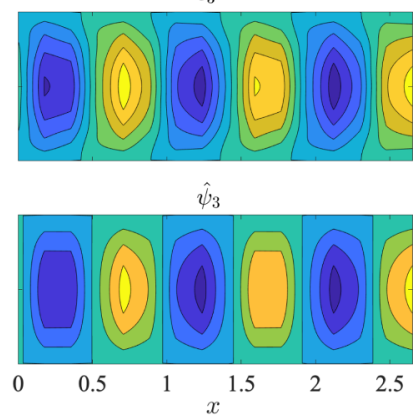

FIG. 4. Temporal Fourier components 1, 2, and 3 for traveling wave solution of thermosolutal convection at $r=2.5$. Fourier components of temperature $\hat{\theta}$, concentration $\hat{c}$ and stream function $\hat{\psi}$ are shown. The components are complex, with combinations of real and imaginary part parametrized by a phase. Here, a single choice of temporal or spatial phase is shown.

If, as is more likely, $u_{n \geqslant 2}$ is not zero, but is small, for example, if

$$
\left\|\hat{u}_{n}\right\| \sim \epsilon^{|n|}
$$

as discussed in Ref. [46], then $\mathcal{N}_{1}$ is of order $\epsilon^{3}$, while $i \omega \hat{u}_{1}$ and $\mathcal{L}_{\bar{U}}$ are of order $\epsilon$, so that Eq. (22) is approximately true. [Note that Eq. (23) does not justify neglecting $\mathcal{N}_{n}$ in Eq. (20b) for $n>1$, since in $\omega \hat{u}_{n}, \mathcal{L}_{\bar{U}} \hat{u}_{n}$, and $\mathcal{N}_{n}$ are all of order $\epsilon^{n}$.]

The argument in terms of spectra is supported by the results of Turton et al. [10]. We recall that standing waves are produced at the same Hopf bifurcation as the traveling waves and that the RZIF property does not hold for the standing waves. In Ref. [10], it is shown that the spectrum of the standing waves is far less peaked at $n=1$ than that of the traveling waves. For example, at $r=2.5$, the ratio of the Fourier components of the temperature field $\left\|\hat{\theta}_{2}\right\| /\left\|\hat{\theta}_{1}\right\|$ is approximately $10^{-2}$ for the traveling waves and 20 times higher for the standing waves.

To be consistent, the quantitative argument based on Eq. (23) would also call for neglecting terms $\mathcal{N}\left(\hat{u}_{m}, \hat{u}_{-m}\right)$ for $m \geqslant 2$ compared to $\mathcal{N}\left(\hat{u}_{1}, \hat{u}_{-1}\right)$, leading to the SCM. The Fourier interpretation of the SCM is that the limit cycle is represented by a temporal Fourier series, truncated to contain only modes $0(\bar{U})$ and $1\left(\hat{u}_{1}\right)$.

In Fig. 5(a) we visualize the temporal Fourier spectra $\left\|\hat{u}_{n}\right\|$ over the range $r \in[2.05,3]$ and for frequencies $n \in[1,8]$. We normalize by $\left\|\hat{u}_{1}\right\|$ since the RZIF approximation relies on neglecting
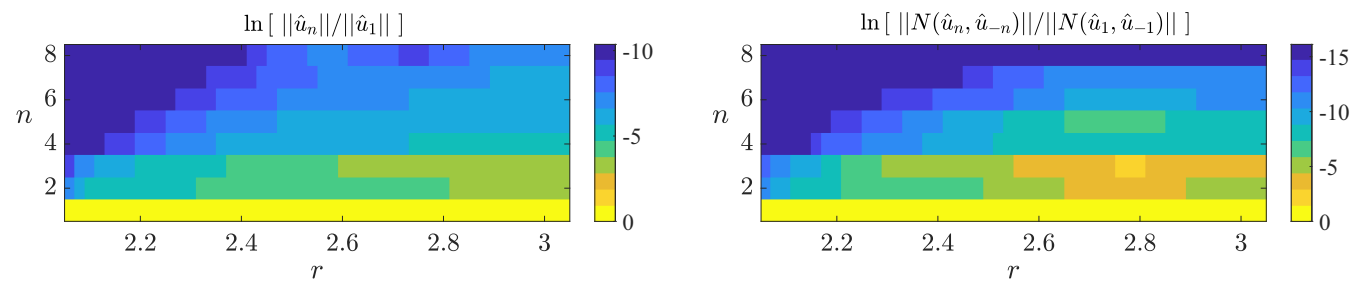

FIG. 5. Logarithmic color representation of Fourier spectra. Left: $\left\|\hat{u}_{n}\right\|$ normalized by $\left\|\hat{u}_{1}\right\|$. Right: Contributions $\left\|\mathcal{N}\left(\hat{u}_{-n}, \hat{u}_{n}\right)\right\|$ to the mean flow normalized by $\left\|\mathcal{N}\left(\hat{u}_{-1}, \hat{u}_{1}\right)\right\|$. 

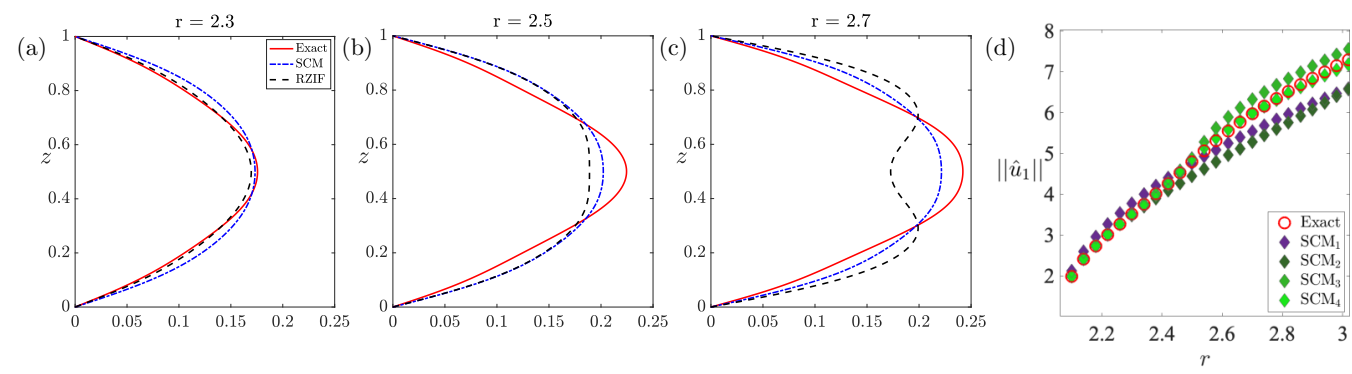

FIG. 6. Modulus of first Fourier component $\left|\hat{c}_{1}(z)\right|$ of concentration field and its approximations via SCM and RZIF for (a) $r=2.3$, (b) $r=2.5$, and (c) $r=2.7$. The amplitudes of the RZIF profiles are undetermined, since they are eigenvectors; here, they have been normalized to match the norms of the Fourier components. SCM overestimates $\left|\hat{c}_{1}\right|$ for $r<2.5$ and underestimates it for $r>2.5$. (d) Norm $\left\|\hat{u}_{1}\right\|$ and its approximation via various orders of SCM as a function of $r$.

$\hat{u}_{n>1}$ in comparison with $\hat{u}_{1}$. Figure 5(b) shows the amplitudes of the nonlinear terms contributing to the mean flow $\left\|\mathcal{N}\left(\hat{u}_{n}, \hat{u}_{-n}\right)\right\|$. We normalize by $\left\|\mathcal{N}\left(\hat{u}_{1}, \hat{u}_{-1}\right)\right\|$, since the SCM assumes that $\mathcal{N}\left(\hat{u}_{n}, \hat{u}_{-n}\right)$ can be neglected in comparison with $\mathcal{N}\left(\hat{u}_{1}, \hat{u}_{-1}\right) \|$. These figures show that both spectra are highly peaked for small $r$ and become less so as $r$ increases, as is to be expected. Going from $n=2$ to 3 , the magnitudes decrease very little, and even increase for higher values of $r$, a point that will be explored further in the next section.

According to Eq. (22), the RZIF procedure does not merely approximate the nonlinear frequency as the leading eigenvalue but also approximates the first temporal Fourier component via the corresponding eigenvector. Figures 6(a)-6(c) illustrate this idea by comparing $\left|\hat{c}_{1}(z)\right|$ with its approximations via RZIF and SCM. Since $c_{\text {rzif }}$ is part of an eigenvector, its norm has been chosen to match that of $\hat{c}_{1}$, i.e., $\int d z\left|c_{\text {rzif }}(z)\right|=\int d z\left|\hat{c}_{1}(z)\right|$. For $r<2.5$, the SCM profile slightly exceeds $\left|\hat{c}_{1}\right|$, while for $r>2.5$ it underestimates it. At $r=2.7$, the $\left|c_{\text {rzif }}\right|$ profile has a secondary minimum which is absent from the corresponding $\left|\hat{c}_{1}\right|$ as well as from $\left|\theta_{\text {rzif }}\right|,\left|\psi_{\text {rzif }}\right|$, $\left|\hat{\theta}_{1}\right|$, and $\left|\hat{\psi}_{1}\right|$. (The secondary minimum is, however, found in $\left|\hat{c}_{1}\right|$ when $L$ is increased to 0.2.) Figure 6(d) compares $\left\|\hat{u}_{1}\right\|=\left[\int d z\left(\left|\hat{t}_{1}(z)\right|^{2}+\left|\hat{c}_{1}(z)\right|^{2}+\left|\hat{\psi}_{1}(z)\right|^{2}\right)\right]^{1 / 2}$ to its SCM approximation, including its higher-order generalizations to be described in the next section. We again see the overestimate by SCM of $\left|\hat{u}_{1}\right|$ for $r<2.5$ and its underestimate for $r>2.5$.

\section{HIGHER-ORDER METHODS}

We have seen that for the traveling waves of thermosolutal convection, linearization about the full mean flow (RZIF) succeeds in matching the frequency of the nonlinear waves, while linearization about a first-order approximation to the mean flow (SCM) does not. It seems natural to consider whether higher-order approximations to the mean flow can lead to a better match.

\section{A. Higher-order SCM}

The SCM is a truncation of the Fourier decomposition of the exact system Eqs. (20a) and (20b) including only components with $|n| \leqslant 1$. A natural idea is to truncate at the next order, $|n| \leqslant 2$. Meliga [16] called this approximation second-order SCM and implemented it for the flow over an open cavity, using a multiple scale expansion method. Truncating at this order, we obtain

$$
\begin{aligned}
0 & =\mathcal{L} \bar{U}+\mathcal{N}(\bar{U}, \bar{U})+\mathcal{N}\left(u_{1}, u_{-1}\right)+\mathcal{N}\left(u_{-1}, u_{1}\right)+\mathcal{N}\left(u_{2}, u_{-2}\right)+\mathcal{N}\left(u_{-2}, u_{2}\right), \\
i \omega u_{1} & =\mathcal{L}_{\bar{U}} u_{1}+\mathcal{N}\left(u_{2}, u_{-1}\right)+\mathcal{N}\left(u_{-1}, u_{2}\right) \\
2 i \omega u_{2} & =\mathcal{L}_{\bar{U}} u_{2}+\mathcal{N}\left(u_{1}, u_{1}\right)
\end{aligned}
$$



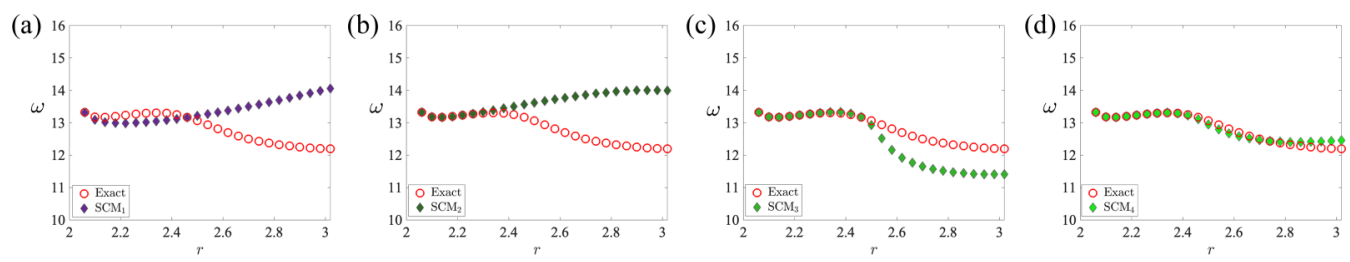

FIG. 7. Frequency calculated by SCM methods of increasing order as a function of Rayleigh number. Exact frequencies are shown by open circles (o), while those predicted by the SCM are shown by diamonds: (a) first $\operatorname{order}(\downarrow)$, (b) second order $(\downarrow)$, (c) third order $(\downarrow)$, and (d) fourth order $(\downarrow)$.

along with a phase condition (see Sec. VI). This system has as unknowns one real $(\bar{U})$ and two complex fields $\left(u_{1}, u_{2}\right)$ and one unknown frequency $(\omega)$. In these equations, $\bar{U}$ does not signify the exact mean flow and the $u_{n}$ 's do not signify the exact Fourier components $\hat{u}_{n}$ but approximations to them. We call this truncated system $\mathrm{SCM}_{2}$. We can also extend Eq. (24) to include higher-order terms, forming third and higher order SCM approximations by truncating the exact representation Eqs. (20a) and (20b) at order $M$ :

$$
\begin{aligned}
& 0=\mathcal{L} \bar{U}+\mathcal{N}(\bar{U}, \bar{U})+\sum_{1 \leqslant|m| \leqslant M} \mathcal{N}\left(u_{m}, u_{-m}\right), \\
& \operatorname{in} \omega u_{n}=\mathcal{L}_{\bar{U}} u_{n}+\sum_{\substack{1 \leqslant|m| \leqslant M \\
1 \leqslant|n-m| \leqslant M}} \mathcal{N}\left(u_{m}, u_{n-m}\right), \quad 1 \leqslant n \leqslant M .
\end{aligned}
$$

Higher-order SCM does not fit into the category of the quasilinear or semilinear models, since nonlinear interactions between $\left\{u_{1}, u_{2}, \ldots\right\}$ that do not contribute to $\bar{U}$ are included, i.e., they are present in Eqs. (24b), (24c), and (25b). Instead, higher-order SCM, like harmonic balance, consists of a consistent truncation in temporal modes at increasingly higher order. The optimal forcing problem for a flat-plate boundary layer was solved at successively higher orders of temporal frequency by Ref. [27].

We solve system Eq. (24) or Eq. (25) by a straightforward Newton's method (see Sec. VI). In these equations (and only here) we have been imprecise in our notation; in theory, $\bar{U}, u_{n}$, and $\omega$ should all carry labels indicating that they are solutions of the $M$ th-order system $\mathrm{SCM}_{\mathrm{M}}$, but such labels would make these equations unreadable.

Figure 7 extends Fig. 2 by comparing the frequencies computed by the higher-order SCM systems with the exact frequencies. Figure 7(b) shows that $\mathrm{SCM}_{2}$ extends the range in which the frequency is well predicted from [2.05, 2.08] to [2.05, 2.3], above which $\mathrm{SCM}_{2}$ increasingly overestimates the frequency. $\mathrm{SCM}_{3}$ extends the matching range up to $r \approx 2.5$, as shown in Fig. 7(c), and underestimates the frequency above this range. Figure $7(\mathrm{~d})$ shows that $\mathrm{SCM}_{4}$ considerably improves the frequency prediction throughout the $r$ range [2.05,3]. Since the $\mathrm{SCM}_{M}$ equations converge to the exact equations with increasing $M$, the corresponding frequencies must converge to the exact frequencies.

Figure 8 extends Fig. 3 by presenting the error in the mean concentration profiles computed by $\mathrm{SCM}_{M}$ as $M$ is increased. Figure 8 (a) at $r=2.3$ shows the dramatic improvement in the mean profile as $M$ is increased past 1, as expected by comparing Figs. 7(a) and 7(b). In contrast, Fig. 8(b) at $r=2.4$ shows that the deviation is as large for the $\mathrm{SCM}_{2}$ profile (and in the opposite direction) as it is for $\mathrm{SCM}_{1}$. Figure 8(c) at $r=2.5$ shows that, rather than improving the profile, the $\mathrm{SCM}_{2}$ approximation is even poorer than that of $\mathrm{SCM}_{1}$. The higher-order profiles converge to the correct profile, but nonmonotonically. This trend continues for $r=2.6$, shown in Fig. 8(d). 

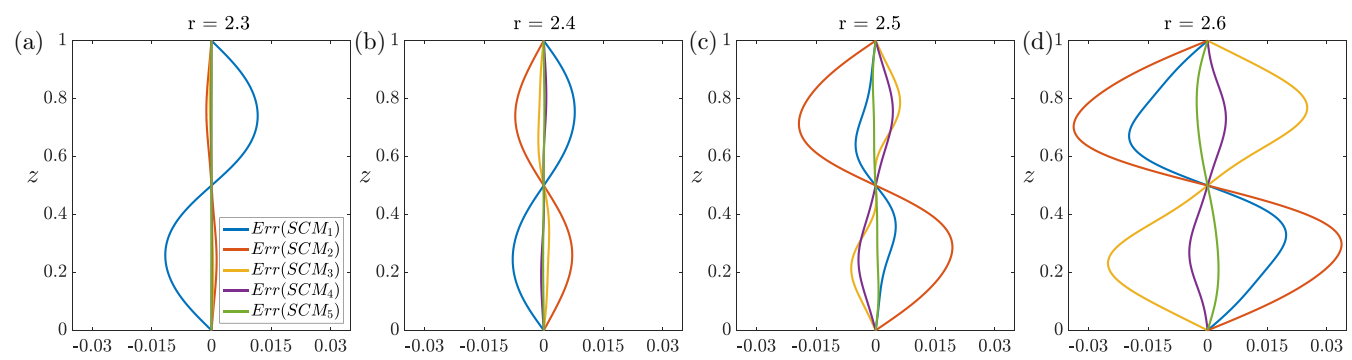

FIG. 8. Mean concentration profiles calculated by various orders of the SCM method compared to exact mean concentration profile. (a) For $r=2.3$, (b) $r=2.4$, (c) $r=2.5$, and (d) $r=2.6$.

\section{B. Incomplete RZIF}

The uneven performance of SCM has motivated us to perform another numerical experiment, namely, to build up the exact mean field by truncating the contributions to it from the exact Fourier coefficients. We denote these approximate mean fields by $\bar{U}_{1}, \bar{U}_{2}, \bar{U}_{3}, \ldots$ and linearize about them:

$$
\begin{aligned}
0 & =\mathcal{L} \bar{U}_{M}+\mathcal{N}\left(\bar{U}_{M}, \bar{U}_{M}\right)+\sum_{1 \leqslant|m| \leqslant M} \mathcal{N}\left(\hat{u}_{m}, \hat{u}_{-m}\right), \\
\left(\sigma_{M}+i \omega_{M}\right) u_{M} & =\mathcal{L}_{\bar{U}_{M}} u_{M},
\end{aligned}
$$

where the $\hat{u}_{m}$ contributing to the mean $\bar{U}_{M}$ in Eq. (26a) are the exact Fourier components of the nonlinear limit cycle defined in Eq. (19). In our case, $\mathcal{N}\left(\bar{U}_{M}, \bar{U}_{M}\right)=\mathcal{N}(\bar{U}, \bar{U})=0$, so Eq. (26a) can be solved via

$$
\bar{U}_{M}=-\mathcal{L}^{-1} \sum_{1 \leqslant|m| \leqslant M} \mathcal{N}\left(\hat{u}_{m}, \hat{u}_{-m}\right)
$$

We will call this the incomplete RZIF approximation.

It is useful to compare this system with the higher-order $\mathrm{SCM}_{M}$ system Eqs. (25a) and (25b) and with the exact system Eqs. (20a) and (20b). Although Eq. (26a) resembles Eq. (25a), we emphasize that the exact Fourier components $\hat{u}_{n}$ of the nonlinear limit cycle $U_{\mathrm{lc}}$ are used in Eq. (26a), as they are in the corresponding exact Eq. (20a). In contrast, the SCM Eq. (25a) uses approximate Fourier components defined self-consistently by the coupled truncated system Eqs. (25a) and (25b). However, Eq. (26b) omits all of the terms $\mathcal{N}_{1}, \mathcal{N}_{2}, \ldots$, as in RZIF, whereas Eq. (25b) includes increasingly accurate versions of these terms. Thus, the incomplete RZIF approximation Eqs. (26a) and (26b) is a gradual approach to RZIF rather than to the full exact Eqs. (20a) and (20b). The incomplete RZIF approximations of various orders are less accurate than the original RZIF method of Secs. II and III, in contrast to the $\mathrm{SCM}_{M}$ methods of various orders, which are more accurate than the original $\mathrm{SCM}_{1}$ method.

Figure 9 shows the eigenvalues resulting from the incomplete RZIF approximation. First, Fig. 9(a) shows the real parts $\sigma_{M}$ as a function of $r$. For $r<2.5, \sigma_{M} \approx 0$, but for $r>2.5$ and for $M=1$ and $M=2$, the values of $\sigma_{M}$ are quite far from zero. Note that $\sigma_{2} \approx \sigma_{1}$, implying that adding the contribution from $\mathcal{N}\left(\hat{u}_{2}, \hat{u}_{2}\right)$ does not improve the estimated mean flow $\bar{U}_{2}$. This is also true for the imaginary parts: $\omega_{2} \approx \omega_{1}$. In Fig. $9(\mathrm{~b})$, we compare $\omega_{2}$ to the exact frequency $\omega_{\mathrm{lc}}$, the RZIF frequency $\omega_{\text {rzif }}$, and the frequency from $\mathrm{SCM}_{2}$. We see that $\omega_{1}$ (not shown in the figure) and $\omega_{2}$ are fairly accurate for $r \leqslant 2.6$, whereas the frequency from $\mathrm{SCM}_{2}$ is accurate only for $r \leqslant 2.4$. For $M=3$, Fig. 9(c) shows that the frequencies $\omega_{3}$ are almost indistinguishable from $\omega_{\mathrm{rzif}}$ and $\omega_{\mathrm{lc}}$, while those from $\mathrm{SCM}_{3}$ still deviate for $r \geqslant 2.5$. Note that $\omega_{M}$ cannot exceed the accuracy of $\omega_{\text {rzif }}$, since the terms $\mathcal{N}_{1}, \mathcal{N}_{2}$, etc. continue to be neglected. This is emphasized in the enlargement of Fig. 9(d), where $\omega_{3}$ is very close to $\omega_{\text {rzif }}$ while remaining apart from $\omega_{\text {lc }}$. For $r>2.4$, the frequency from $\mathrm{SCM}_{4}$ follows a different trend. 

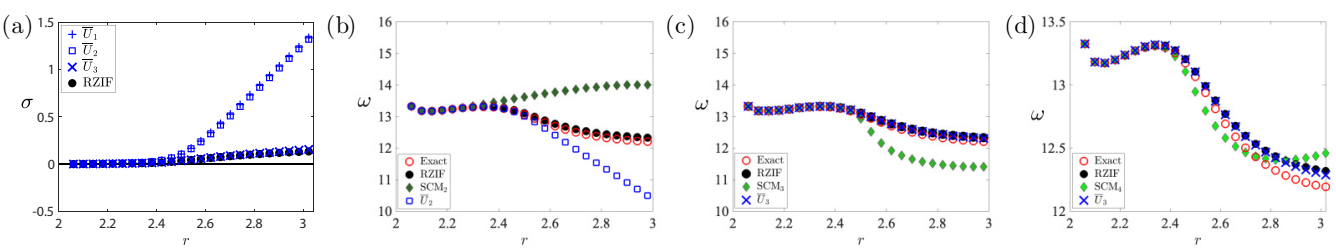

FIG. 9. Comparison of the incomplete RZIF and SCM methods. (a) Growth rates calculated by linearizing about the full mean (RZIF) are represented by solid black circles $(\bullet)$. Those obtained by linearizing about $\bar{U}_{1}, \bar{U}_{2}$, and $\bar{U}_{3}$ are represented by blue plus signs ( + ), by blue crosses $(\times)$, and by hollow blue boxes $(\square)$, respectively. The growth rates from $\bar{U}_{1}$ and $\bar{U}_{2}$ are almost indistinguishable, as are the growth rates from $\bar{U}_{3}$ and RZIF. (b, c, d) Frequencies. Exact frequencies are represented by open red circles (o) while those obtained by linearizing about the full mean field (RZIF) are represented by solid black circles $(\bullet)$. Those obtained by linearizing about $\bar{U}_{2}$ and $\bar{U}_{3}$ are represented by hollow blue boxes $(\square)$, and by blue crosses $(\times)$, respectively. Frequencies predicted by the SCM are shown by diamonds: (b) second order $(\downarrow)$, (c) third order $(\downarrow)$, and (d) fourth order $(\diamond)$. Linearization about $\bar{U}_{3}$ achieves the same results as RZIF, so no further improvement is possible. $\mathrm{SCM}_{4}$ is not as accurate.

Figure 10 shows the error in the mean concentration profiles resulting from successively truncating the Fourier series, as in Eq. (26a). These errors are considerably smaller than the corresponding errors from the SCM analysis; the scale of Fig. 10 is a third of that of Fig. 8. We see that going from $\bar{U}_{1}$ to $\bar{U}_{2}$ does not substantially decrease the error in the incomplete RZIF approximation, while $\bar{U}_{3}$ achieves the accuracy of RZIF, as was seen in Fig. 9 for the eigenvalues.

The incomplete RZIF approximation removes the effect of approximating the Fourier components, leaving only the effect of truncating the Fourier sum. The less satisfactory performance of SCM compared to the incomplete RZIF method of the same order can thus be attributed to the inaccuracy in SCM's estimates of $\hat{u}_{1}, \hat{u}_{2}, \ldots$, leading to inaccuracy in the estimated mean flow. Including higher-order modes produced by self-consistent truncations proves less successful than including their exact versions at the same order.

We mention that neither of the families of methods-higher-order SCM nor incomplete RZIFfall precisely into the category of QL or GQL methods. We recall that QL or GQL methods divide the modes into two types, the mean (or low frequency) modes and the other (or high frequency) modes. One set of equations involves only the projections onto low modes of low-low or high-high quadratic terms. The other set involves only mixed low-high quadratic terms, so that the high frequency modes obey equations which are linear in the high frequency terms. In contrast, the RZIF methods use externally calculated (exact) fields while the SCM methods include all interactions between the retained modes.
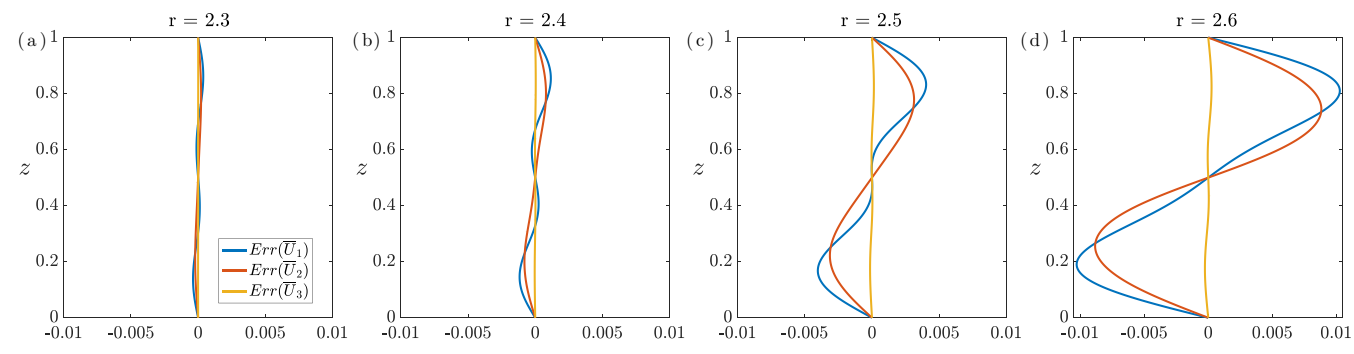

FIG. 10. Mean concentration profiles calculated by various orders of the incomplete RZIF compared to exact mean concentration profile. (a) For $r=2.3$, (b) $r=2.4$, (c) $r=2.5$, and (d) $r=2.6$. Note that the scale of the horizontal axis is one third that of Fig. 8, indicating greater accuracy for the results of the incomplete RZIF method compared to the SCM. 


\section{ALGORITHMS}

\section{A. Thermosolutal convection}

We first describe the methods particular to thermosolutal convection. The spatial discretization consists of a Fourier series in the periodic direction $x$ and a sine series in the vertical direction $z$ (allowed for the streamfunction because of the free-slip boundaries). Differentiation is carried out in Fourier-sine space and multiplication in the grid space. For our parameter range and boundary conditions, very little resolution is needed; the $(x, z)$ rectangle is represented by a $16 \times 8$ grid. By defining

$$
U \equiv(\Theta, C, \Psi)^{T},
$$

we rewrite Eq. (17) in the compact notation used previously,

$$
\partial_{t} U=\mathcal{L} U+\mathcal{N}(U, U) .
$$

We carry out time evolution by a mixed scheme, in which diffusive terms $\mathcal{L}$ are evolved via the implicit Euler method and the remaining terms by the explicit Euler method,

$$
U(t+\Delta t)=(I-\Delta t \mathcal{L})^{-1}\{U(t)+\Delta t \mathcal{N}[U(t), U(t)]\} .
$$

When the limit cycle $U_{\mathrm{lc}}$ is a traveling wave, it is a stationary state in a moving reference frame governed by

$$
V \partial_{x} U_{\mathrm{lc}}=\mathcal{L} U_{\mathrm{lc}}+\mathcal{N}\left(U_{\mathrm{lc}}, U_{\mathrm{lc}}\right)
$$

where $V=\lambda / T_{\mathrm{lc}}=\omega_{\mathrm{lc}} / k$ is the wave speed, with $\lambda$ the wavelength, $T_{\mathrm{lc}}$ the period, $k$ the wave number, and $\omega_{\mathrm{lc}}$ the angular frequency. The term $V \partial_{x} U$ can be moved to the right-hand-side and integrated explicitly along with $\mathcal{N}$. The traveling waves are computed via Newton's method by transforming Eq. (30) as described in Ref. [10], with time stepping providing initial estimates for fields and wave speeds. Compensating for the additional variable of the wave speed $V$, a phase condition such as

$$
\partial_{x} \widetilde{U}_{\mathrm{lc}}(x=0)=0
$$

is imposed, where $\widetilde{U}$ is one of $\Theta, C, \Psi$ at a fixed value of $z$. The traveling wave solution is continued from one value of $r$ to the next to cover the range [2.06,3].

When the limit cycle is not a traveling wave, as is the case for the cylinder wake or the standing waves of thermosolutal convection, it must be calculated via time integration. Another possibility is to use Newton's method with shooting to redefine the limit cycle as a fixed point problem in a much higher dimensional space.

\section{B. RZIF and SCM systems}

We now discuss algorithmic aspects specific to the RZIF and SCM equations. For RZIF, the limit cycle solution is averaged over time (or equivalently, for a traveling wave, over the $x$ direction) to produce $\bar{U}$. The Jacobian about $\bar{U}$ is computed and diagonalized to produce its leading eigenvalue $\sigma_{\text {rzif }}+i \omega_{\text {rzif }}$. For this small problem, matrix operations such as diagonalization and inversion for Newton's method can be carried out directly, but for larger problems, matrix-free iterative methods such as BiCGSTB, GMRES, or IDR and the Arnoldi or power methods can be used.

We now turn to the SCM:

$$
\begin{aligned}
0 & =\mathcal{L} U_{\mathrm{scm}}+\mathcal{N}\left(U_{\mathrm{scm}}, U_{\mathrm{scm}}\right)+\mathcal{N}\left(u_{\mathrm{scm}}, u_{\mathrm{scm}}^{*}\right), \\
i \omega_{\mathrm{scm}} u_{\mathrm{scm}} & =\mathcal{L}_{U_{\mathrm{scm}}} u_{\mathrm{scm}},
\end{aligned}
$$

together with a phase condition. The unknowns are the real field $U_{\mathrm{scm}}$, the complex field $u_{\mathrm{scm}}$, and the scalar $\omega_{\text {scm. }}$. We solve the coupled system Eqs. (33a) and (33b) via a straightforward Newton's 
method. We start near the threshold $r=r_{\text {Hopf }}$, where $U_{\mathrm{scm}}=U_{\mathrm{b}}$ (which is zero in the thermosolutal case) and $u_{\mathrm{scm}}=u_{\mathrm{b}}, \omega_{\mathrm{scm}}=\omega_{\mathrm{b}}$. For higher $r$ values, the initial estimate used is the solution at the previous value of $r$.

Mantič-Lugo et al. $[13,14]$ solve the SCM equations by an iterative algorithm that decouples the two equations. Equation (33a) is treated as a nonlinear equation for $U_{\mathrm{scm}}$ with $\mathcal{N}\left(u_{\mathrm{scm}}, u_{\mathrm{scm}}\right)$ as an inhomogeneous forcing term, while Eq. (33b) is treated as an eigenproblem with fixed $U_{\text {scm }}$ defining the linear operator. As it stands, Eq. (33b) is not an eigenproblem, since $\mathcal{L}_{U_{\text {scm }}}$ is expected to have complex eigenvalues rather than pure imaginary ones. (The closely related operator $\mathcal{L}_{U_{\mathrm{b}}}$ has an imaginary eigenpair only exactly at the Hopf bifurcation.) In addition, Eq. (33b) does not fix a normalization for $u_{\mathrm{scm}}$, which is required for $\mathcal{N}\left(u_{\mathrm{scm}}, u_{\mathrm{scm}}\right)$ when it is used as an input for Eq. (33a). Such considerations lead these authors to specify a norm $A$ for $u_{\mathrm{scm}}$ (or, equivalently, to multiply a normalized $u_{\mathrm{scm}}$ by $A$ ). Equation (33b) is replaced by

$$
\begin{gathered}
\left(\sigma_{\mathrm{scm}}+i \omega_{\mathrm{scm}}\right) u_{\mathrm{scm}}=\mathcal{L}_{U_{\mathrm{scm}}} u_{\mathrm{scm}}, \\
\left\|u_{\mathrm{scm}}\right\|=A
\end{gathered}
$$

where $\sigma_{\text {scm }}$ and Eq. (33d) are an additional unknown and equation relative to Eq. (33b), while $A$ is an input value.

Determining $U_{\mathrm{scm}}, u_{\mathrm{scm}}$, and $\omega_{\mathrm{scm}}$ for a single value of $r$ requires looping over values of $A$ as follows. $A$ is initially set to zero, since then Eqs. (33a) and (33c) are the equations governing the base flow and leading eigenpair from classical linear stability analysis; their solution is $U_{\mathrm{b}}, \sigma_{\mathrm{b}}+i \omega_{\mathrm{b}}$, $u_{\mathrm{b}}$. To solve the equations for a new $A>0, u_{\mathrm{scm}}$ is given norm $A$ and substituted into Eq. (33a) to generate a new $U_{\text {scm }}$, which is in turn substituted into Eqs. (33c) and (33d), leading to a new $u_{\mathrm{scm}}$ that is substituted into Eq. (33a). The process is continued until $U_{\mathrm{scm}}, \sigma_{\mathrm{scm}}+i \omega_{\mathrm{scm}}$, and $u_{\mathrm{scm}}$ cease to change. $A$ is then increased and the procedure repeated, using as initial estimates the solutions for the previous $A$. The calculation is halted and the solution accepted when a value of $A$ is reached for which $\sigma_{\mathrm{scm}}=0$. Thus, the real-zero portion of the RZIF hypothesis is built into the method.

However, even if Eqs. (33a) and (33c), (33d) can be individually satisfied, there is no guarantee of convergence of the coupled system for a given $A$. Nor is it guaranteed that there will be a value of $A$ such that $\sigma(A)=0$. When Mantič-Lugo et al. $[13,14]$ used the decoupled algorithm to compute the SCM approximation for the cylinder wake, they reported convergence problems, in response to which they introduced a relaxation factor and a different normalization of $\mathcal{N}\left(u_{\mathrm{scm}}, u_{\mathrm{scm}}\right)$ to improve convergence; more details about the algorithm can be found in Refs. [14,47]. With these modifications, they were then able to accurately reproduce the frequency of the cylinder wake for Reynolds numbers up to $\operatorname{Re}=120$.

Meliga [16] implemented the second-order $\mathrm{SCM}_{2}$ given by Eq. (24) by generalizing the approach in Refs. [13,14], writing a series of nested sub-problems for $\bar{U}, u_{1}, u_{2}$, and two auxiliary complex fields, each solved via Newton's method and the Arnoldi method. As in Refs. [13,14], an amplitude $A$ was imposed and the solution was considered to be reached when a growth rate reached zero.

In our case of traveling waves in thermosolutal convection, we were able to use the decoupled algorithm Eq. (33a) and Eqs. (33c), (33d) for $r$ only $2 \%$ above $r_{\text {Hopf }}$; above this value, the decoupled algorithm does not converge. In contrast, the full Newton method performed robustly for Eqs. (33a) and (33b), as well as for the higher-order SCM system Eqs. (25a) and (25b). We note that Fani et al. [15] also applied a full Newton method to solve the SCM for the acoustic generation of the Bénard-von Kármán vortex street, using MUMPS to solve the large sparse linear system required by Newton's method. We have presented the coupled algorithm for several reasons:

(i) We wished to make contact with the literature.

(ii) Our thermosolutal problem is quite small. Although our method is considerably simpler, it is possible that a decoupled method such as that in Refs. [13,14] or Ref. [16] might be needed for a larger problem. 
(iii) The decoupled algorithm has the advantage of describing the amplitude saturation process, mimicking the evolution of $A$ in time, discussed in Refs. [48,49]. The unstable base field solution extracts energy from the perturbations, which grow until they saturate. SCM computes the mean field, the nonlinear frequency and the nonlinear mode along with its amplitude $A$ without time integration.

\section{CONCLUSION}

Nonlinear equations can be interpreted as governing the coupled evolution of modes, canonically Fourier modes. Various truncations have been proposed to either speed up computations or to gain a greater understanding of the behavior of their solutions. A basic task, which may be considered to be a benchmark of such truncations, is to match the frequency of a limit cycle.

RZIF consists of computing the temporal mean, linearizing the evolution operator about it, and then calculating its leading eigenvalue [6]. This approximation has been shown to be resoundingly successful in the archetypal case of the wake of the circular cylinder [6], the traveling waves of thermosolutal convection [10], the ribbons and spirals of counter-rotating Taylor-Couette flow [11], and the shear-driven flow over a square cavity [12]. Although RZIF has thus far been applied only to limit cycles produced by supercritical Hopf bifurcations, it is plausible that it might also apply when the bifurcations are subcritical, since the mean upon which it relies is obtained from the nonlinear limit cycle, independently of its distance from the base flow. The search for a general reason for this success is constrained by the existence of a clear counterexample: the standing waves of thermosolutal convection that bifurcate at the same parameter value as the traveling waves [10]. Based on this counterexample, Turton et al. [10] proposed that the dominance of the primary Fourier mode could serve as a criterion for success of RZIF, which pushes the question further upstream to when and why the primary Fourier mode dominates.

RZIF confers theoretical insight but no practical advantages, since the temporal mean is calculated from a full simulation of the limit cycle. For this reason, Mantič-Lugo et al. [13,14] proposed to close the equations by limiting them to the mean flow and the primary temporal Fourier mode and showed that this SCM method succeeded as dramatically as RZIF on the archetypal cylinder wake. However, Fig. 2 shows that the traveling waves of thermosolutal convection that satisfy the RZIF property so well cannot be approximated by the SCM. Although the interaction between higher-order modes may be omitted from the higher-order Eqs. (20b), their contribution to the mean flow remains important: they cannot be removed from Eq. (20a) governing the mean flow. In addition, in our example, a good approximation of the mean flow requires that the higher-order modes contributing to it be accurately represented, as demonstrated by Figs. 7 and 9. From this example, it would seem to be interactions, rather than modes, that can be omitted. However, other examples, e.g., Ref. [21], argue in the opposite direction.

Despite verifying RZIF, the traveling waves of our thermosolutal convection problem verify SCM in a very narrow interval around one parameter value and not elsewhere. The thermosolutal standing waves that provide a counter example to RZIF are generated at precisely the same bifurcation and with the same parameter values as the traveling waves. These counter examples provide a warning that truncations must be carefully controlled and understood, and that doing so may prove unexpectedly difficult.

From the example of the cylinder wake [13,14] and its compressible version [15] as well as the shear-driven cavity [16], it is clear that the SCM works remarkably well even for fairly complex hydrodynamic problems, while yielding a major reduction in calculation costs. The challenge is to determine which configurations are amenable to SCM and why.

[1] H. Bénard, Formation périodique des centres de giration à l'arrière d'un obstacle en mouvement, C. R. Acad. Sci. Paris 147, 839 (1908). 
[2] T. von Kármán, Über den Mechanismus des Widerstandes, den ein bewegter Körper in einer Flüssigkeit erfährt, Nachr. K. Ges. Wiss. Gött., Math.-Phys. Kl. 5, 509 (1911).

[3] C. Jackson, A finite-element study of the onset of vortex shedding in flow past variously shaped bodies, J. Fluid Mech. 182, 23 (1987).

[4] M. Provensal, C. Mathis, and L. Boyer, Bénard-von Kármán instability: Transient and forced regimes, J. Fluid Mech. 182, 1 (1987).

[5] B. Pier, On the frequency selection of finite-amplitude vortex shedding in the cylinder wake, J. Fluid Mech. 458, 407 (2002).

[6] D. Barkley, Linear analysis of the cylinder wake mean flow, Europhys. Lett. 75, 750 (2006).

[7] D. Sipp and A. Lebedev, Global stability of base and mean flows: A general approach and its applications to cylinder and open cavity flows, J. Fluid Mech. 593, 333 (2007).

[8] S. Mittal, Global linear stability analysis of time-averaged flows, Int. J. Numer. Methods Fluids 58, 111 (2008).

[9] W. Malkus, Outline of a theory of turbulent shear flow, J. Fluid Mech. 1, 521 (1956).

[10] S. E. Turton, L. S. Tuckerman, and D. Barkley, Prediction of frequencies in thermosolutal convection from mean flows, Phys. Rev. E 91, 043009 (2015).

[11] Y. Bengana and L. S. Tuckerman, Spirals and ribbons in counter-rotating Taylor-Couette flow: Frequencies from mean flows and heteroclinic orbits, Phys. Rev. Fluids 4, 044402 (2019).

[12] Y. Bengana, J.-C. Loiseau, J.-C. Robinet, and L. Tuckerman, Bifurcation analysis and frequency prediction in shear-driven cavity flow, J. Fluid Mech. 875, 725 (2019).

[13] V. Mantič-Lugo, C. Arratia, and F. Gallaire, Self-Consistent Mean Flow Description of the Nonlinear Saturation of the Vortex Shedding in the Cylinder Wake, Phys. Rev. Lett. 113, 084501 (2014).

[14] V. Mantič-Lugo, C. Arratia, and F. Gallaire, A self-consistent model for the saturation dynamics of the vortex shedding around the mean flow in the unstable cylinder wake, Phys. Fluids 27, 074103 (2015).

[15] A. Fani, V. Citro, F. Giannetti, and F. Auteri, Computation of the bluff-body sound generation by a selfconsistent mean flow formulation, Phys. Fluids 30, 036102 (2018).

[16] P. Meliga, Harmonics generation and the mechanics of saturation in flow over an open cavity: A secondorder self-consistent description, J. Fluid Mech. 826, 503 (2017).

[17] B. McKeon and A. Sharma, A critical-layer framework for turbulent pipe flow, J. Fluid Mech. 658, 336 (2010).

[18] B. J. McKeon, A. Sharma, and I. Jacobi, Experimental manipulation of wall turbulence: A systems approach, Phys. Fluids 25, 031301 (2013).

[19] Y. Hwang and C. Cossu, Linear nonnormal energy amplification of harmonic and stochastic forcing in the turbulent channel flow, J. Fluid Mech. 664, 51 (2010).

[20] V. Mantic-Lugo and F. Gallaire, Self-consistent model for the saturation mechanism of the response to harmonic forcing in the backward-facing step flow, J. Fluid Mech. 793, 777 (2016).

[21] V. Mantič-Lugo and F. Gallaire, Saturation of the response to stochastic forcing in two-dimensional backward-facing step flow: A self-consistent approximation, Phys. Rev. Fluids 1, 083602 (2016).

[22] S. Beneddine, D. Sipp, A. Arnault, J. Dandois, and L. Lesshafft, Conditions for validity of mean flow stability analysis, J. Fluid Mech. 798, 485 (2016).

[23] S. Beneddine, R. Yegavian, D. Sipp, and B. Leclaire, Unsteady flow dynamics reconstruction from mean flow and point sensors: An experimental study, J. Fluid Mech. 824, 174 (2017).

[24] S. Symon, K. Rosenberg, S. T. M. Dawson, and B. J. McKeon, Nonnormality and classification of amplification mechanisms in stability and resolvent analysis, Phys. Rev. Fluids 3, 053902 (2018).

[25] S. Symon, D. Sipp, and B. J. McKeon, A tale of two airfoils: Resolvent-based modelling of an oscillator versus an amplifier from an experimental mean, J. Fluid Mech. 881, 51 (2019).

[26] E. Yim, P. Meliga, and F. Gallaire, Self-consistent triple decomposition of the turbulent flow over a backward-facing step under finite amplitude harmonic forcing, Proc. R. Soc. A 475, 20190018 (2019).

[27] G. Rigas, D. Sipp, and T. Colonius, Nonlinear input/output analysis: Application to boundary layer transition, J. Fluid Mech. 911, A15 (2021). 
[28] D. Gayme, B. McKeon, A. Papachristodoulou, B. Bamieh, and J. Doyle, A streamwise constant model of turbulence in plane Couette flow, J. Fluid Mech. 665, 99 (2010).

[29] V. L. Thomas, B. K. Lieu, M. R. Jovanović, B. F. Farrell, P. J. Ioannou, and D. F. Gayme, Self-sustaining turbulence in a restricted nonlinear model of plane Couette flow, Phys. Fluids 26, 105112 (2014).

[30] F. Alizard and D. Biau, Restricted nonlinear model for high-and low-drag events in plane channel flow, J. Fluid Mech. 864, 221 (2019).

[31] D. F. Gayme and B. A. Minnick, Coherent structure-based approach to modeling wall turbulence, Phys. Rev. Fluids 4, 110505 (2019).

[32] E. Yim, P. Billant, and F. Gallaire, Nonlinear evolution of the centrifugal instability using a semilinear model, J. Fluid Mech. 897, A34 (2020).

[33] K. Rosenberg and B. J. McKeon, Computing exact coherent states in channels starting from the laminar profile: A resolvent-based approach, Phys. Rev. E 100, 021101(R) (2019).

[34] M. Pausch, Q. Yang, Y. Hwang, and B. Eckhardt, Quasilinear approximation for exact coherent states in parallel shear flows, Fluid Dyn. Research 51, 011402 (2019).

[35] J. B. Marston, G. P. Chini, and S. M. Tobias, Generalized Quasilinear Approximation: Application to Zonal Jets, Phys. Rev. Lett. 116, 214501 (2016).

[36] B. F. Farrell and P. J. Ioannou, Structure and spacing of jets in barotropic turbulence, J. Atmos. Sci. 64, 3652 (2007).

[37] K. Srinivasan and W. Young, Zonostrophic instability, J. Atmos. Sci. 69, 1633 (2012).

[38] S. M. Tobias and J. B. Marston, Direct Statistical Simulation of Out-of-Equilibrium Jets, Phys. Rev. Lett. 110, 104502 (2013).

[39] S. M. Tobias and J. B. Marston, Three-dimensional rotating Couette flow via the generalized quasilinear approximation, J. Fluid Mech. 810, 412 (2017).

[40] A. Allawala, S. M. Tobias, and J. B. Marston, Dimensional reduction of direct statistical simulation, J. Fluid Mech. 898, A21 (2020).

[41] L. S. Tuckerman, Thermosolutal and binary fluid convection as a $2 \times 2$ matrix problem, Physica D 156, 325 (2001).

[42] E. Knobloch, Oscillatory convection in binary mixtures, Phys. Rev. A 34, 1538 (1986).

[43] K. C. Hall, J. P. Thomas, and W. S. Clark, Computation of unsteady nonlinear flows in cascades using a harmonic balance technique, AIAA J. 40, 879 (2002).

[44] M. McMullen, A. Jameson, and J. Alonso, Demonstration of nonlinear frequency domain methods, AIAA J. 44, 1428 (2006).

[45] M. S. McMullen and A. Jameson, The computational efficiency of nonlinear frequency domain methods, J. Comput. Phys. 212, 637 (2006).

[46] J. Dušek, P. Le Gal, and P. Fraunié, A numerical and theoretical study of the first Hopf bifurcation in a cylinder wake, J. Fluid Mech. 264, 59 (1994).

[47] V. Mantič Lugo, Too big to grow: self-consistent model for nonlinear saturation in open shear flows, Ph.D. thesis, Ecole Polytechnique Fédérale de Lausanne, Lausanne (2015).

[48] A. Maurel, V. Pagneux, and J. Wesfreid, Mean-flow correction as nonlinear saturation mechanism, Europhys. Lett. 32, 217 (1995).

[49] B. J. A. Zielinska, S. Goujon-Durand, J. Dusek, and J. E. Wesfreid, Strongly Nonlinear Effect in Unstable Wakes, Phys. Rev. Lett. 79, 3893 (1997). 